\title{
Emodialisi domiciliare intensiva: uno sguardo al passato alla ricerca dell'emodialisi del futuro
}

\author{
Agostino Naso, Giuseppe Scaparrotta, Maria Loreta De Giorgi, Gianni Carraro
}

\author{
UOC di Nefrologia 2, DIMED, Azienda Ospedaliera di Padova, Padova
}

\begin{abstract}
INTENSIVE HOME HEMODIALYSIS: AN EYE AT THE PAST AND LOOKING FOR THE HEMODIALYSIS OF THE FUTURE
Abstract. The results of many observational studies and some randomized clinical trials seem to point toward intensive home hemodialysis (IHD) as being able to improve the outcome of uremic patients under chronic dialysis with a cost/benefit ratio more favorable than conventional hemodialysis.

The increasing interest in home hemodialysis in the last years is due to its identification as the easiest strategic option for the realization of programs of intensive hemodialysis.

However, a careful attention has to be paid in the selection of candidate patients for home hemodialysis; specifically, the psychosocial, demographic, and clinical factors are some of the aspects that need a major consideration due to their impact on the success of such programs. Further studies are strongly needed to better clarify the clinical ground and the limits of IHD use in dialysis patients.
\end{abstract}

Key words: Uremia, Hemodialysis, Home hemodialysis, Short daily hemodialysis, Nocturnal-extended hemodialysis, NxStage

Conflict of interest: None.

Financial support: None.

Accettato: 1 Settembre 2014

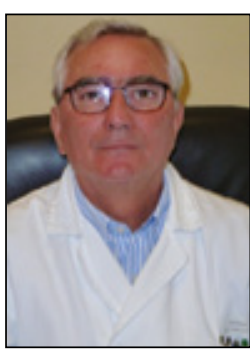

Agostino Naso

Il primo programma europeo di emodialisi domiciliare (DHD) è stato attivato in Inghilterra nei primi anni ' 60 del secolo scorso da Shaldon, con lo scopo di aumentare le possibilità di trattamento in un'epoca di scarsa disponibilità di posti dialisi e di crescente fabbisogno dialitico. Negli stessi anni, DePalma pubblicava il primo studio su 7 pazienti trattati con dialisi di 5 sedute settimanali di 4-5 ore, uno schema di terapia dialitica intensiva, straordinariamente innovativo, se confrontato con quello di pochi anni prima, quando la dialisi era lunga e intermittente, con sessioni di 12-18 ore ogni 10-15 giorni in assenza di condizioni urgenti (1). Negli anni '70-'80, i progressi nel campo dialitico e i buoni risultati clinici ottenuti con l'introduzione di dializzatori ad alta efficienza portarono a una progressiva riduzione della durata delle sedute dialitiche, con l'affermarsi dello schema dialitico standard (CHD) di 4 ore x 3/settimana, che consentiva un maggiore utilizzo dei posti dialisi, nel frattempo aumentati per la diffusione sempre più capillare dei Centri dialisi. Si assisteva così, a partire dalla seconda metà degli anni '80, a un continuo declino della DHD, a cui non erano estranei la nascita dei Centri ad Assistenza Limitata, i cambiamenti intervenuti nelle caratteristiche cliniche e demografiche della po- polazione dialitica e il ricorso crescente alla dialisi peritoneale come terapia domiciliare dell'uremia. La DHD, dopo una fase di ascesa che la portò a rappresentare, negli anni '70, il 40\% della popolazione dialitica negli USA (2) e il 38-50\% dei dializzati in alcuni Centri dialisi del nord Italia (3), divenne, a dispetto dei benefici clinici e sociali riportati, un'infrequente modalità di terapia sostitutiva dell'uremia.

Dalla fine della prima decade del 2000 a oggi, è stata registrata negli USA un'inversione di tendenza nel ricorso alla DHD, con un aumento dei pazienti da 4511 nel 2009 a oltre 6000 nel 2012 (4). Il rinnovato interesse nei confronti della metodica è dovuto sia ai progressi intervenuti nel campo della tecnologia dialitica sia a ragioni di ordine clinico.

I primi hanno consentito di disporre di apparecchiature disegnate "ad hoc" per la DHD, facili da usare, con tempi di apprendimento di appena 4-8 settimane e con circuiti preassemblati e tempi di priming e restituzione rapidi, così da ridurre significativamente l'impegno temporale della singola seduta. Una delle nuove apparecchiature, la NxStage, può utilizzare soluzioni di dialisi in sacche, ovviando alla necessità di opere idrauliche al domicilio del paziente per la preparazione dell'acqua di dialisi; impiegata, poi, con i parametri operativi indicati (Tab. I) permette di ottenere saturazioni del dialisato per l'urea vicine al 100\%, con l'uso di appena 20-25 L di soluzione/seduta. 
TABELLA I - NXSTAGE: PARAMETRI OPERATIVI

\begin{tabular}{lc}
\hline Sedute/sett. (n.) & $5-6$ \\
Durata seduta (ore) & $2.5-3.5$ \\
$\mathrm{Qb}(\mathrm{mL} / \mathrm{min})$ & $300-450$ \\
$\mathrm{Qd}(\mathrm{mL} / \mathrm{min})$ & $83-200$ \\
$\mathrm{Calcio}(\mathrm{mmol} / \mathrm{L})$ & 1.5 \\
Tampone dialisato & Lattato, $40-45 \mathrm{mmol} / \mathrm{L}$ \\
Dialisato/seduta (L) & $20-30$ \\
Potassio (mmol/L) & $1.0-2.0$ \\
$\mathrm{D} / \mathrm{P} \mathrm{Urea}$ & $\sim 100 \%$ \\
$\mathrm{Qd} / \mathrm{Qb}$ & $\sim 1 / 3$ \\
\hline
\end{tabular}

TABELLA II - MODALITÀ DI TERAPIA DIALITICA INTENSIVA

\begin{tabular}{lcccc}
\hline & CHD & SDHD & NHD & NxStage HD \\
\hline Sedute/sett. (n.) & 3 & $5-6$ & $5-6$ & $5-6$ \\
Durata seduta (ore) & 4 & $2-3$ & $6-8$ & $2.5-3.5$ \\
Qb (mL/min) & 400 & 400 & 200 & $300-400$ \\
Qd (mL/min) & 500 & 800 & 300 & $100-130$ \\
sKt/V & 1.3 & 0.5 & 1.8 & $0.4-0.7$ \\
\hline
\end{tabular}

CHD: dialisi convenzionale; SDHD: dialisi breve giornaliera; NHD: dialisi notturna (o lunga); NxStage HD: dialisi breve giornaliera con NxStage.

Le ragioni cliniche della rinascita sono da ricondurre invece alla capacità della DHD di superare le barriere logisticoorganizzative che si oppongono all'attuazione di programmi di dialisi intensiva (IHD) (Tab. II), verso cui si guarda oggi con grande interesse per migliorare l'elevata mortalità della popolazione dialitica, specie dopo i deludenti risultati ottenuti dall'HEMO Study (5). Fino a oggi l'IHD con aumento della frequenza e/o della durata delle sedute era prevista dalle Linee Guida europee (6) come rescue therapy nei casi di grave ipertensione arteriosa refrattaria, grave instabilità emodinamica, grave malnutrizione o inadeguato controllo di elevati livelli di fosforo sierico. Le indicazioni erano suggerite dagli eccellenti risultati ottenuti dal Centro di Tassin in Francia nella sopravvivenza a 10 anni e nel controllo pressorio in oltre 800 pazienti trattati in 40 anni con dialisi di 8 ore $x$ 3/settimana e da Buoncristiani in Italia e Ting negli USA con la SDHD. La prima applicazione dell'IHD sotto forma di dialisi domiciliare notturna (NHD) al di fuori delle indicazioni cliniche sopra menzionate si deve a Uldall, che la attuò nel 1994 nell'ambito di un programma sponsorizzato dal Ministero della Salute dell'Ontario. Dal Canada la NHD si diffuse in molti altri Paesi, tra cui gli USA, dove, ad opera di Lockridge, venne varato il più largo programma di NHD (1).

Alla fine del 1990, il gruppo di Uldall riportava i dati relativi all'esperienza maturata con la NHD nell'arco di 3 anni (7). In particolare, il report riguardava i risultati clinici e biochimici ottenuti in 12 pazienti dializzati a domicilio con sessioni di 8-10 ore per 6-7 notti/settimana, utilizzando come accesso vascolare un $\mathrm{CVC}$ in giugulare interna, con $\mathrm{Qb}$ di $300 \mathrm{~mL} / \mathrm{min}$ e Qd di 100 mL/min; confrontata con la CHD, la NHD determinava una rimozione doppia di fosfati e 4 volte più elevata di $\beta 2$ microglobulina, con sospensione dei chelanti del fosforo nonostante un aumentato introito dietetico di proteine. Vi era anche un miglioramento del controllo pressorio con riduzione degli ipotensivi, mentre le complicanze erano infrequenti e riconducibili soprattutto all'accesso vascolare.

Nel 1997, fu pubblicato il primo trial randomizzato controllato (8) sugli effetti della NHD vs la CHD sull'IVS (end point primario) e su qualità della vita, controllo pressorio, metabolismo minerale e consumo di farmaci (end point secondari); erano reclutati 52 pazienti, randomizzati in rapporto $1: 1$ alla NHD domiciliare o alla CHD. Comparata con la CHD, la NHD migliorava significativamente l'IVS e alcuni parametri della qualità della vita e del metabolismo minerale e riduceva il consumo di ipotensivi.

Nel 2010 e nel 2011, il "Frequent Hemodialysis Network" pubblicava i risultati di 2 importanti trial, FHN-Daily Trial e FHN-Nocturnal Trial, 2 studi randomizzati controllati supportati dal NIH $(9,10)$. Nel Daily Trial, 125 e 120 pazienti erano rispettivamente randomizzati per 12 mesi alla SDHD in centro e alla CHD. I 2 principali outcome compositi dello studio erano la morte o le variazioni dell'IVS e la morte o le variazioni del PHC score-RAND-36. Gli end point secondari includevano la performance cognitiva, la depressione, i marker di metabolismo minerale, l'anemia, la pressione arteriosa, il grado di ospedalizzazione e gli interventi sull'accesso vascolare.

Entrambi gli end point compositi erano significativamente migliori nei soggetti assegnati alla SDHD, che si associava anche a un migliore controllo pressorio e dell'iperfosforemia, ma vi erano più interventi sull'accesso vascolare nei pazienti sottoposti a dialisi frequente.

Nel FHN Nocturnal Trial, in cui 45 e 42 pazienti erano rispettivamente assegnati per 12 mesi alla NHD domiciliare e alla CHD, non vi era invece alcuna differenza significativa per quanto riguardava gli stessi end point compositi principali del Daily Trial, mentre era confermato il miglioramento della pressione sistolica e del fosforo sierico predialitici. I risultati ottenuti dallo studio erano interpretati come probabilmente dovuti alla scarsa numerosità della casistica, alla minore aderenza alla terapia dialitica nei pazienti assegnati alla NHD, con circa $1 / 3$ dei pazienti che effettuavano meno dell' $80 \%$ delle sedute prescritte e all'elevata prevalenza di uremici incidenti $(>50 \%)$ con la possibilità che la funzione renale residua avesse attenuato $\mathrm{i}$ benefici potenzialmente associati alla dialisi frequente. A conferma di quest'ultima ipotesi, ci sembra di un certo interesse l'osservazione che nel FHN Daily Trial, diversamente che nel Nocturnal Trial, si aveva una riduzione significativa dei volumi ventricolari e che questa fosse particolarmente accentuata nei soggetti con diuresi residua $<100 \mathrm{~mL} /$ die $(11)$.

I benefici sul sistema cardiovascolare promossi dall'IHD erano confermati da una recente metanalisi (12) e da uno studio com- 
parativo condotto in soggetti sottoposti a dialisi di crescente intensità, valutati mediante ecocardiografie quantitative in serie, finalizzate a studiare il numero di alterazioni intradialitiche della motilità ventricolare sinistra. I pazienti assegnati alla SDHD presentavano meno stunning miocardico e marker più favorevoli di danno cardiaco (13).

Un altro aspetto clinico a cui si guarda con grande interesse è quello relativo ai possibili vantaggi ottenibili con la dialisi frequente sulla qualità della vita (QOL), le cui misure risultano fortemente deficitarie con la CHD. In un Report ad interim del "The NxStage Freedom Study", uno studio osservazionale in corso negli USA che ha lo scopo di registrare i benefici clinici ed economici promossi dalla SDHD, era segnalato a 12 mesi un miglioramento di diverse componenti della salute psicofisica, valutate con SF-36 e con un raddoppio della percentuale di soggetti con PCS score equivalente alla popolazione normale (14). Non meno importanti i risultati di altre analisi ad interim, provenienti ancora dallo studio FREEDOM, che evidenziavano a 12 mesi una riduzione dal $41 \%$ al $27 \%$ dei pazienti con sintomi depressivi, una riduzione dell'astenia post dialitica, dei disturbi del sonno e della prevalenza e della gravità della restless legs syndrome $(15,16)$.

Sembrerebbe pertanto che la dialisi intensiva si associ a favorevoli risultati su alcuni surrogati di outcome cardiovascolare e alcuni indicatori della qualità della vita. Resta invece da chiarire se gli apparenti benefici clinici si associno a un'aumentata sopravvivenza. A tale proposito disponiamo solo di studi osservazionali, gravati purtroppo da un insieme di bias di difficile accettazione in un momento di Medicina delle Evidenze basata prevalentemente su trial clinici randomizzati.

In un report riguardante 415 pazienti sottoposti a SDHD per 1006 anni/paziente era segnalata una sopravvivenza a 5 anni del $68 \%$ e a 10 anni del $42 \%, 2-3$ volte più elevata rispetto alla popolazione dialitica statunitense di confronto e sovrapponibile a quella di trapiantati da cadavere (CDTX) della stessa età (17). L'assenza di differenza nella sopravvivenza tra IHD e CDTX era confermata da uno studio canadese relativo a 177 pazienti sottoposti a NHD (3-7 sessioni/sett.; 6-8 h/sessione) confrontati con una popolazione di uremici dell'USRDS (18), ma non in uno studio più recente, proveniente da un singolo centro canadese che, comparando sopravvivenza/fallimento della terapia e ospedalizzazione tra 173 pazienti in IHD (tempo di trattamento $\geq 16$ ore/settimana) e circa 1500 trapiantati dello stesso centro, riportava una riduzione del rischio di mortalità/fallimento della terapia di circa il $60 \%$ nei trapiantati, con una riduzione, invece, del rischio di ospedalizzazione nei dializzati nel primo anno di terapia (19). Il vantaggio di sopravvivenza dell'IHD era confermato ma in misura minore in alcuni studi recenti condotti con metodi statistici rigorosi allo scopo di attenuare l'impatto negativo del bias di selezione e correggendo per un insieme di variabili cliniche di significato prognostico (20-22).

Assolutamente inaspettata perciò è giunta una nuova analisi dei dati dell'International Quotidian Dialysis Registry (23) che, in controcorrente rispetto a un'analisi precedente (21), tratta da dati dello stesso registro, riportava un aumento del rischio di mortalità del $60 \%$ con la SDHD, ponendo l'attenzione sui possibili rischi associati alla più frequente esposi- zione all'unphysiology della dialisi e alle complicanze della dialisi frequente, di cui diremo a breve. Va, però, segnalato che al recente congresso dell'EDTA di Amsterdam è stato comunicato che dopo un follow up medio di 3.6 anni nei pazienti randomizzati alla dialisi frequente nel FHN Daily Trial vi era una riduzione del rischio di mortalità del $46 \%$, nonostante il ritorno alla CHD alla fine dello studio (24).

L'emodialisi intensiva non è priva di complicanze. In entrambi i trial del FHN era riportata un'aumentata incidenza di interventi sull'accesso vascolare che riguardava oltre il $50 \%$ dei soggetti randomizzati alla NHD e il $38 \%$ di quelli randomizzati alla SDHD, contro rispettivamente il $36 \%$ e il $24 \%$ dei pazienti randomizzati alla CHD. È difficile non mettere in relazione i problemi riguardanti l'accesso vascolare con il suo più frequente utilizzo, inconveniente non eliminabile a meno di non ricorrere a tecniche meno traumatiche di puntura della FAV. Aveva perciò suscitato un grande interesse tra i nefrologi uno studio randomizzato e controllato che riportava una maggiore sopravvivenza della FAV con la puntura buttonhole, con meno interventi sull'accesso e meno complicanze aneurismatiche (25). I vantaggi sulla durata dell'accesso vascolare non erano però confermati da una recente review, che ne sottolineava invece l'aumentato rischio di infezioni (26).

Il FHN Nocturnal Trial, a differenza del Daily Trial, era complicato anche da una più rapida perdita della funzione renale residua, probabilmente riconducibile al migliorato controllo dei volumi extracellulari promosso dall'allungamento del tempo di dialisi, ma da considerare con attenzione alla luce del ruolo oggi ad essa attribuito per la preservazione dell'architettura del ventricolo sinistro e il miglioramento della sopravvivenza $(27,28)$.

Un oggettivo ostacolo alla diffusione su più larga scala dell'IHD è la possibile scarsa compliance alla terapia dialitica, che comportava nel NHD-FHN la riduzione delle sessioni dialitiche a meno dell' $80 \%$ di quelle prescritte nel $30 \%$ dei casi e che, anche nella nostra limitata esperienza, è stata causa in 1 caso del fallimento della metodica. L'osservazione va considerata una spia del sempre presente rischio di burn-out del paziente e/o caregiver e della necessità di una scelta accurata nella selezione dei pazienti da avviare a un programma di IHD, tenendo conto dei numerosi e diversi fattori in gioco nel determinismo del successo o del fallimento della metodica. In uno studio recente dei determinanti del fallimento del training o della DHD si indicava nel $16 \%$ dei casi la causa nei fattori psicosociali con, al primo posto, l'abitare in una casa in affitto, mentre tra $\mathrm{i}$ fattori clinici in ordine decrescente di rischio vi erano il diabete, il CVC come accesso vascolare, l'indice di Charlson e l'età (29). L'importanza di una selezione attenta di pazienti è indirettamente richiamata da un'analisi di costoutilità di un recente studio canadese, che sottolinea come $\mathrm{i}$ maggiori benefici a costi più bassi della NHD verso la CHD siano persi e tendano a invertirsi quando il fallimento della metodica è superiore al $7.6 \%(30)$.

In conclusione, l'IHD può migliorare l'outcome degli uremici in dialisi, ma la sua diffusione comporta problemi di ordine economico, logistico e organizzativo più facilmente superabili con il ricorso alla DHD.

Le innovazioni tecnologiche nel campo delle apparecchiature 
dialitiche consentono oggi di superare alcune delle barriere che ostacolano la diffusione della DHD, ma l'IHD non è una dialisi adatta a tutti i pazienti.

Nuovi studi e osservazioni saranno necessari per definire il campo clinico della sua applicazione e per guidarci nella scelta dell'opzione dialitica giusta per il paziente giusto.

\section{Riassunto}

Negli ultimi anni vi è stato un rinnovato interesse verso la dialisi domiciliare, che appare come la strategia operativa più facilmente percorribile per l'attuazione di programmi di emodialisi intensiva (IHD). Numerosi studi osservazionali e pochi studi randomizzati controllati fanno ritenere che l'IHD possa migliorare l'outcome degli uremici in dialisi con un rapporto costi/benefici migliore dell'emodialisi convenzionale.

È necessaria tuttavia un'attenta valutazione nella selezione dei pazienti da avviare all'IHD, alla luce dei fattori psicosociali, clinici e demografici che possono determinarne il fallimento. Ulteriori studi saranno necessari per definire i limiti e il campo clinico di applicazione dell'IHD.
Parole chiave: Uremia, Emodialisi, Emodialisi domiciliare, Emodialisi breve giornaliera, Emodialisi notturna o lunga, NxStage

Dichiarazione di conflitto di interessi: Gli Autori dichiarano di non avere conflitto di interessi.

Contributi economici agli Autori: Gli Autori dichiarano di non aver ricevuto sponsorizzazioni economiche per la preparazione dell'articolo.

Indirizzo degli Autori:

Dr. Agostino Naso

Via A. Gabelli 35

35128 Padova

agostino.naso@sanita.padova.it

\section{Bibliografia}

1. Perl J, Chan CT. Home Hemodialysis, Daily Hemodialysis, and Nocturnal hemodalysis: Core Curriculum 2009. Am J Kidney Dis. 2009; 54 (6): 1171-84.

2. Blagg CR. A brief history of home hemodialysis. Adv Ren Replace Ther. 1996; 3: 99-105.

3. Lombardi M. Ha ancora senso fare emodialisi domiciliare nel 2001? Giornale di Tecniche Nefrologiche \& Dialitiche 2001; 3 (Suppl. 2): S5-8.

4. Young BA, Chan C, Blagg C, et al. How to overcome barriers and establish a successful home HD program. Clin J Am Soc Nephrol. 2012; 7: 2023-4.

5. Eknoyan G, Beck GJ, Cheung AK, et al. Effect of dialysis dose and membrane flux in maintenance hemodialysis. N Engl J Med . 2002; 347 (25): 2010-9.

6. Tattersal J, Martin-Malo A, Pedrini L, et al. EBPG guideline on dialysis strategies. Nephrol Dial Transplant. 2007; 22 (Suppl. 2): 5-21.

7. Pierratos A, Ouendyk M, Francoeur R, et al. Nocturnal hemodialysis: Three-Year Experience. J Am Soc Nephrol. 1998; 9: 859-68.

8. Culleton BF, Walsh M, Klarenbach SW, et al. Effect of frequent nocturnal hemodialysis vs conventional hemodialysis on left ventricular mass and quality of life: a randomized controlled trial. JAMA. 2007; 298: 1291-9.

9. The FHN trial Group. In-Center hemodialysis six times per week versus three times per week. N Engl Med. 2010; 363 (24): 2287-300.

10. Rocco MV, Lockridge RS, Beck GJ, et al. The effects of frequent nocturnal home hemodialysis: the frequent hemodialysis network nocturnal trial. Kidney Int. 2011; 80: 1080-91.

11. Chan CT, Greene T,Chertow GM, et al. Effects of frequent hemodialysis on ventricular volumes and left ventricular remodeling. Clin J Am Soc Nephrol. 2013; 8: 2106-16.

12. Susantitaphong P, Koulouridis I, Balk EM, Madias NE,Jaber BL. Effect of frequent or extended hemodialysis on cardiovascular parameters: a meta-analysis. Am J Kidney Dis. 2012; 59 (5): 689-99.

13. Jefferies HJ, Virk B, Schiller B, Moran J, McIntyre CW. Frequent hemodialysis schedules are associated with reduced levels of dialysis-induced cardiac Injury(myocardial stunning). Clin J Am Soc Nephrol. 2011; 6: 1326-32.

14. Finkelstein FO, Schiller B, Daoui R, et al. At-home short daily hemodialysis improves the long term health-related quality of life. Kidney Int. 2012; 82: 561-9.

15. Jaber BL, Lee Y, Collins AJ, et al. Effects of daily hemodialysis on depressive symtoms and postdialysis recovery time: interim report from the FREEDOM (Following Rehabilitation, Economics and Everyday-Dialysis Outcome) Study. Am J Kidney Dis. 2010; 56: 531-9.

16. Jaber BL, Schiller B, Burkart JM, et al. Impact of short daily hemodialysis on restless legs symptoms and sleep disturbance. Clin J Am Soc Nephrol. 2011; 6: 1049-56.

17. Kjellstrand CM, Buoncristiani U, Ting G, et al. Short daily hemodialysis: serviva in 415 patients treated for 1006 patientyears. Nephrol Dial Tranplant. 2008; 23: 3283-9.

18. Pauly RP, Gill JS, Rose CS, et al. Survival among nocturnal home haemodialysis compared to kidney transplant patients. Nephrol Dial Tranplant. 2009; 24: 2915-9.

19. Tennankore KK, Kim SJ, Baer HJ, Chan CT. Survival and hospitalization for intensive home hemodialysis compared with kidney 
transplantation. J Am Soc Nephrol. 2014; 25 (9): 2113-20.

20. Weinhandl ED, Liu J, Gilbertson DT, Arneson TJ, Collins AJ. Survival in daily hemodialysis and matched thrice-weekly in-center hemodialysis patients. J Am Soc Nephrol. 2012; 23: 895-904.

21. Nesrallah GE, Lindsay RM, Cuerden MS, et al. Intensive hemodialysis associates with improved survival compared with conventional hemodialysis. J Am Soc Nephrol. 2012; 23: 696-705.

22. Lacson E, Xu J, Suri RS, et al. Survival with three-times weekly in-center nocturnal versus conventional hemodialysis. J Am Soc Nephrol. 2012; 23: 687-95.

23. Suri RS, Lindsay RM,Bieber BA, et al. A multinational cohort study of in-center daily hemodialysis and patient survival. Kidney Int. 2013; 83: 300-7.

24. Kliger AS, Chertow GM, Levin NW, et al. Long-term effects of frequent in-center hemodialysis: FHN Daily TRIAL. Nephrol Dial Transplant. 2014; 29 (Suppl. 3): iii37-8.

25. Vaux E, King J, LloidS, et al. Effect of buttonhole cannulation with a polycarbonate peg on in-center hemodialysis fistula outcomes: a randomized controlled trial. Am J Kidney Dis. 2013; 62 (1): 81-8.

26. Muir AM, Kotwal SS, Hawley CM, et al. Buttonhole cannulation and clinical outcomes in a home hemodialysis cohort and Systematic Review. Clin J Am Soc Nephrol. 2014; 9: 110-9.

27. Daugirdas JT, Green T, Rocco MV, et al. Effect of frequent hemodialysis on residual kidney function. Kidney Int. 2013; 83 (5): 949-58.

28. Vilar E, Farrington K. Emerging importance in residual renal function in end-stage renal failure. Semin Dial. 2011; 24: 487-94.

29. Schachter ME, Tennankore KK, Chan CT. Determinants of training and technique failure in Home hemodialysis. Hemodial Int. 2013; 17 (3): 421-6.

30. Klarer B, Tonelli M, Pauly R, et al. Economic evaluation of frequent home nocturnal hemodialysis based on a randomized controlled trial. J Am Soc Nephrol. 2014; 25: 587-94. 